\title{
Preliminary research on the effects and mechanisms of umbilical cord-derived mesenchymal stem cells in streptozotocin-induced diabetic retinopathy
}

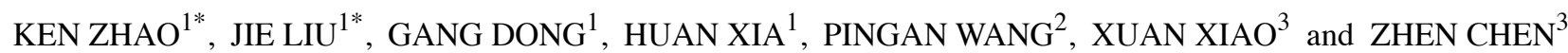 \\ ${ }^{1}$ Department of Ophthalmology, People's Hospital of Daye, The Second Affiliated Hospital of Hubei Polytechnic College, \\ Daye, Hubei 435100; ${ }^{2}$ Wuhan Myhalic Biotechnology Co., Ltd., Wuhan, Hubei 430206; \\ ${ }^{3}$ Department of Ophthalmology, Renmin Hospital of Wuhan University, Wuhan, Hubei 430060, P.R. China
}

Received October 24, 2019; Accepted April 16, 2020

DOI: $10.3892 /$ ijmm.2020.4623

\begin{abstract}
Diabetic retinopathy (DR) is one of the most prevalent microvascular complications of diabetes, and a common cause of blindness in working-age individuals. Mesenchymal stem cell (MSC) transplantation has been considered a promising intervention therapy for DR, wherein the differentiation of MSCs into nerve cells plays an essential role. However, research into the role of MSCs in DR treatment remains incomplete, and the mechanisms of retinal repair at the molecular level have yet to be clarified. In the present study, all-trans retinoic acid (ATRA) was used to promote the proliferation of rat umbilical cord (UC)-derived MSCs and their differentiation into nerve cells. Furthermore, the effects and mechanisms of UC-MSCs with or without ATRA treatment were investigated in rats subjected to streptozocin (STZ)-induced DR. The results demonstrated that the transplantation of UC-MSCs treated with or without ATRA attenuated DR in rats, and alleviated retinal tissue damage and apoptosis. In addition, the transplantation of UC-MSCs treated with or without ATRA
\end{abstract}

Correspondence to: Dr Xuan Xiao or Dr Zhen Chen, Department of Ophthalmology, Renmin Hospital of Wuhan University, Zhang Road (formerly Ziyang Road), 99 Jiefang Road 238, Wuchang, Wuhan, Hubei 430060, P.R. China

E-mail: xiaoxuan1111@163.com

E-mail: hchenzhen@163.com

${ }^{*}$ Contributed equally

Abbreviations: DR, diabetic retinopathy; UC-MSCs, umbilical cord-derived mesenchymal stem cells; ATRA, all-trans retinoic acid; STZ, streptozocin; NES, neuron-specific enolase; GFAP, glial fibrous acidic protein; CTGF, connective tissue growth factor; t-PA, tissue plasminogen activator; PAI, plasminogen activator inhibitor; TGF $\beta 2$, transforming growth factor $\beta 2$; VEGF, vascular endothelial growth factor; IL-1, interleukin-1

Key words: umbilical cord-derived mesenchymal stem cells, transplantation, diabetic retinopathy, angiogenesis, inflammation attenuated angiogenesis and inflammation in the retina by regulating the levels of relevant cytokines. UC-MSCs treated with ATRA exerted a more prominent therapeutic effect than the untreated UC-MSCs. On the whole, these findings indicate that UC-MSCs alleviate STZ-induced DR in rats by regulating angiogenesis and the inflammatory response at the molecular level. Thus, the findings of the present study may provide a theoretical basis for the application of MSCs in the treatment of DR.

\section{Introduction}

Diabetic retinopathy (DR) is a primary microvascular complication of diabetes mellitus. DR is characterized by inflammation and neuronal dysfunction associated with concomitant abnormal angiogenesis that leads to inevitable and permanent visual deficits (1). Epidemiological and clinical research has revealed that the global diabetic population in 2011 was approximately 350 million and this is estimated to increase to approximately 500 million by the year 2030 (2), one-third of which will suffer from DR (3). Thus, numerous therapies have been proposed to alleviate retinopathy induced by hyperglycemia, including vitrectomy, laser photocoagulation and the intravitreal injection of anti-vascular endothelial growth factor (VEGF) agents (4). Although the aforementioned interventions have exhibited notable therapeutic effects in the treatment of DR, there are some inevitable side-effects, such as peripheral vision loss, decrease in night vision and the loss of visual sensitivity (5).

Mesenchymal stem cells (MSCs) are stromal cells that have the ability to self-renew and also exhibit multilineage differentiation potential (6). Owing to their high expandability in vitro and their excellent ability to differentiate, MSCs are considered to be a promising resource for stem cell-based therapy (7). Recent studies have demonstrated that MSC transplantation, or a combination with other factors, has broad application prospects in the treatment of DR $(8,9)$. The results of animal experiments have also demonstrated that the transplantation of MSCs into the retina of DR-affected rats effectively delayed retinal degeneration through neuroprotection (10). Moreover, MSCs have been shown to improve the integrity 
of the blood-retinal barrier by differentiating into glial-like and photoreceptor cells in the retina, resulting in alleviated DR in rats with streptozotocin (STZ)-induced diabetes (11). Nevertheless, the exact role of MSCs in the treatment of DR has not yet been fully determined, and the mechanisms of retinal repair at the molecular level remain unclear.

With the advantage of procurement, storage and transplantation compared to other stem cells, umbilical cord-derived MSCs (UC-MSCs) have attracted increasing attention in clinical settings $(12,13)$. A previous study suggested that MSC transplantation ameliorated STZ-induced DR in rats, although the reduced viability of MSCs attenuated the therapeutic effect (14). According to previous studies, all-trans retinoic acid (ATRA), a metabolic product of vitamin A (15), and the retinoid signaling pathway are involved in several biological processes, leading to neuronal proliferation and differentiation (16). Additionally, ATRA induces the in vitro differentiation of stem cells into several types of neural cells, such as neuroblastoma (17), embryonic stem (18) and embryonic carcinoma cells (19). It also enhances the migration and proliferation of MSCs, and induces UC-MSC differentiation into neuron-like cells $(20,21)$. Therefore, in the present study, UC-MSCs with or without ATRA treatment were transplanted into the vitreous body of rats subjected to STZ-induced DR. Retinal pathology was observed in terms of inflammation and angiogenesis, which are known to play essential roles in the pathogenesis of DR $(22,23)$. The levels of related cytokines were determined to preliminarily examine the effects and mechanisms of UC-MSCs on STZ-induced DR. The findings of the present study may provide a theoretical basis for and new insight into the application of MSCs in the treatment of DR.

\section{Materials and methods}

Extraction and treatment of UC-MSCs. Sprague-Dawley rats, purchased from the Laboratory Animal Centre, Huazhong Agricultural University, were artificially caged at a male-female ratio of 1:2 to obtain rats at 19 days of gestation. Caesarean section was performed after the rats were euthanized with an intraperitoneal injection of pentobarbital sodium in $150 \mathrm{mg} / \mathrm{kg}$ to collect the umbilical cord tissue, and vascular tissue was subsequently removed. The remaining Wharton's jelly tissue was then cut up and digested with Dulbecco's modified Eagle's medium (DMEM, HyClone; GE Healthcare Life Sciences) containing 0.1\% type IV collagenase (Gibco; Thermo Fisher Scientific, Inc.) for $10 \mathrm{~min}$, followed by $0.25 \%$ trypsinization for $20 \mathrm{~min}$ at $37^{\circ} \mathrm{C}$. Following purification, the cells were resuspended in DMEM containing $1 \%$ penicillin-streptomycin-amphotericin B solution (Bioswamp, Myhalic Biotechnology Co., Ltd.) and 10\% fetal bovine serum (FBS, Gibco; Thermo Fisher Scientific, Inc.) at a concentration of $2 \times 10^{5}$ cells $/ \mathrm{ml}$, seeded into a culture flask pre-coated with polylysine (Sigma-Aldrich; Merck KGaA) and incubated under constant humidity conditions at $37^{\circ} \mathrm{C}$ in an atmosphere containing $5 \% \mathrm{CO}_{2}$. The culture medium was changed the following day and every 3 days thereafter. Passaging was carried out after the cells reached $85 \%$ confluence. UC-MSCs in the third passage were examined for CD29, CD44, CD73, CD105, CD90, CD34, CD45 and HLA-DR expression by flow cytometry (Beckman Coulter, Inc.) and observed under an inverted fluorescence microscope (Leica Microsystems $\mathrm{GmbH}$ ). The obtained UC-MSCs were then treated with $0.5 \mu \mathrm{M}$ ATRA (Shanghai Aladdin Biochemical Technology Co., Ltd.) for 12, 24 or $48 \mathrm{~h}$, as previously described (21). Untreated UC-MSCs served as the control (CON).

3-(4,5-Dimethyl-2-thiazolyl)-2,5-diphenyl-2-H-tetrazolium bromide (MTT) assay. MTT assay was performed to evaluate the viability of the ATRA-treated UC-MSCs. Briefly, $180 \mu \mathrm{l}$ of cells in the logarithmic phase were plated into 96-well plates at a concentration of $5 \times 10^{3}$ cells/well and incubated at constant humidity at $37^{\circ} \mathrm{C}$ in an atmosphere containing $5 \% \mathrm{CO}_{2}$ overnight. Following treatment with ATRA $(0.5 \mu \mathrm{M})$ for 12,24 or $48 \mathrm{~h}$, the cells were treated with $20 \mu \mathrm{l}$ of MTT $(5 \mathrm{mg} / \mathrm{ml}$; Bioswamp, Myhalic Biotechnology Co., Ltd.) for $4 \mathrm{~h}$, followed by the addition of $150 \mu \mathrm{l}$ of dimethyl sulfoxide. The absorbance was measured using a microplate reader (Thermo Fisher Scientific, Inc.) at $490 \mathrm{~nm}$ after 10 min of shaking.

Flow cytometry. Flow cytometry was carried out to identify the UC-MSCs and evaluate the apoptosis of UC-MSCs with or without ATRA treatment. For cell identification, the cells were collected at a concentration of $1 \times 10^{6} \mathrm{cell} / \mathrm{ml}$ into 8 Eppendorf tubes, with $100 \mu \mathrm{l}$ of cells in each tube, wherein $2 \mu 1$ of CD29-fluorescein isothiocyanate (FITC) (85-11-0291-80; eBioscience, Inc.), CD44-FITC (MA5-16906; eBioscience, Inc.), CD90-FITC (554894, BD Biosciences), CD34-FITC (MA1-10204; eBioscience, Inc.), CD45-FITC (11-0460-82; eBioscience, Inc.), CD73-FITC (PAB45829; Bioswamp, Myhalic Biotechnology Co., Ltd.), CD105-FITC (MA1-19231; Invitrogen; Thermo Fisher Scientific, Inc.), or $1 \mu \mathrm{l}$ of HLA-DR antibody (ab175085, Abcam) were added followed by incubation for $45 \mathrm{~min}$ in the dark (one tube acted as the control). After washing with phosphate-buffered saline (PBS, Bioswamp, Myhalic Biotechnology Co., Ltd.), the cells were resuspended with $100 \mu \mathrm{l}$ flow cytometry staining buffer and incubated with $2 \mu \mathrm{l}$ FITC-conjugated Affinipure goat anti-rabbit $\operatorname{IgG}(\mathrm{H}+\mathrm{L})$ (PAB160016; Bioswamp, Myhalic Biotechnology Co., Ltd.) for $45 \mathrm{~min}$ in the dark, $400 \mu \mathrm{l}$ of flow cytometry staining buffer was added. The cells were analyzed, and data were acquired using a flow cytometer (Beckman Coulter, Inc.). For the evaluation of apoptosis, cells were harvested by trypsin, resuspended in a binding buffer at a concentration of $1 \times 10^{5}$ cells $/ \mathrm{ml}$, labeled with $10 \mu \mathrm{l}$ of Annexin V-FITC and $10 \mu \mathrm{l}$ of propidium iodide (PI) for $30 \mathrm{~min}$ at room temperature, and analyzed by flow cytometry. A minimum of $2 \times 10^{4}$ cells was acquired for each sample.

Immunofluorescence. Following treatment with ATRA, the cell status was detected by immunofluorescence. Incubated cells were washed twice with PBS (pH 7.4) and fixed in $4 \%$ paraformaldehyde at room temperature for $30 \mathrm{~min}$, followed by 3 washes in PBS. The cells were then permeabilized using $0.5 \%$ Triton X-100 (Bioswamp, Myhalic Biotechnology Co., Ltd.) in PBS for 20 min and blocked in 5\% bovine serum albumin (BSA) for $1 \mathrm{~h}$. The cells were subsequently incubated with primary antibodies against Nestin (1:200, ab92391, Abcam), neuron-specific enolase (NSE; 1:200, ab53025, Abcam) and glial fibrous acidic protein (GFAP; 1:200, ab16997, Abcam) for $1 \mathrm{~h}$ at room temperature, and thereafter with AlexaFluor 
488-conjugated Affinipure goat anti-rabbit secondary antibody (1:10,000, SAB43742, Bioswamp, Myhalic Biotechnology Co., Ltd.) for $1 \mathrm{~h}$ at room ttemperature. The nuclei were stained with DAPI (Bioswamp, Myhalic Biotechnology Co., Ltd.) and stained cells were examined under a fluorescence microscope (Leica, Microsystems GmbH).

Reverse transcription-quantitative polymerase chain reaction $(R T-q P C R)$. The mRNA expression levels of Nestin, NSE and GFAP in UC-MSCs following treatment with ATRA for $24 \mathrm{~h}$ were evaluated by RT-qPCR. Total RNA was extracted using TRIzol reagent (Ambion) and reversed transcribed into cDNA according to the following system: Total RNA (500 ng) combination with $4 \mu 15 \mathrm{X}$ PrimeScript II buffer (Takara), $2 \mu 1,10 \mathrm{mM}$ of dNTP Mix (Solarbio), $1 \mu 1$ Oligo DT18 primer (Takara), $1 \mu 1,40 \mathrm{U} / \mu \mathrm{l}$ of Recombinant RNase Inhibitor (Takara), $1 \mu \mathrm{l}$,

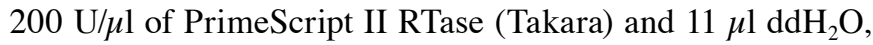
followed by amplification using SYBR FAST qPCR Master Mix (KM4101, Kapa Biosystems). The thermocycling conditions are as follows: $95^{\circ} \mathrm{C}$ for $3 \mathrm{~min} ; 40$ cycles of denaturation at $95^{\circ} \mathrm{C}$ for $50 \mathrm{sec}$, annealing at $56^{\circ} \mathrm{C}$ for $10 \mathrm{sec}$, and extension at $72^{\circ} \mathrm{C}$ for $20 \mathrm{sec}$; and final extension from $65^{\circ} \mathrm{C}$ to $95^{\circ} \mathrm{C}$ at the rate of $5 \mathrm{sec} / 0.5^{\circ} \mathrm{C}$. The primer sequences were as follows: NSE forward, 5'-GCCTCTACGGGCATCT-3' and reverse, 5'-ATC AGGTTGTCCAGTTTC-3'; GFAP forward, 5'-GACGCA TCACCTCCGCT-3' and reverse, 5'-CGCCTTGTTTTGCTG TTC-3'; Nestin forward, 5'-AGGGGCAGACATCATTGG-3' and reverse, 5'-CAGAGACTAGCGGCATTCC-3'; GAPDH forward, 5'-CCACTCCTCCACCTTTG-3' and reverse, 5'-CAC CACCCTGTTGCTGT-3'. The $2^{-\Delta \Delta C q}$ method was utilized to calculate the relative mRNA expression levels (24). GAPDH served as an internal reference gene.

Animal model. A total of 40 Sprague-Dawley rats (weighing $120 \pm 7.0 \mathrm{~g}, 7$ weeks old) were obtained from the Laboratory Animal Centre, Huazhong Agricultural University, and raised in a specific-pathogen-free room with a humidity of 50-60\% and a temperature of $23 \pm 2^{\circ} \mathrm{C}$ with standard water and diet. Diabetes was induced with a single intraperitoneal injection of freshly prepared STZ (Sigma-Aldrich; Merck KGaA) solution in citrate buffer (pH 4.5, Sigma-Aldrich; Merck $\mathrm{KGaA}$ ) at $60 \mathrm{mg} / \mathrm{kg}$ as previously described (22). The rats were confirmed to be diabetic when the fasting blood glucose levels were measured to be $\geq 16.7 \mathrm{mmol} / \mathrm{l}$ at 1 week after the injection, as previously described (25). Diabetic rats were fed a standard diet and unlimited water for 2 months. The fasting blood glucose levels were continuously monitored each week to ensure the models were successful. Insulin was not used during the study for the treatment of any extreme glycemic levels. All animal protocols were approved by the Institutional Review Board of Wuhan Myhalic Biotechnology Co., Ltd. based on the ethical Guidelines for Animal Care and Use of the Model Animal Research Institute (HLK-20180802-01) and were performed in accordance with the Association for Research in Vision and Ophthalmology Statement for the Use of Animals in Ophthalmic and Vision Research.

Intravitreal injection of UC-MSCs in diabetic rats. After 2 months, 30 diabetic rats with lens opacities (milky-white colored lens) were selected to establish the DR model and were randomly divided into 3 groups ( $\mathrm{n}=10$ per group) as follows: The model (MOD), ATRA and UC-MSC group. The rats in the MOD group were intravitreally injected with $2 \mu \mathrm{l}$ of saline. Those in the ATRA and UC-MSC groups were administered an intravitreal injection of ATRA-treated or normal UC-MSCs (2 $\mu 1,1 \times 10^{5-6}$ cells), respectively (12). The control rats (CON group, $n=10$ ) were not subjected to STZ induction. Prior to the injection, UC-MSCs with or without ATRA treatment were labeled with CM-Dil (Invitrogen; Thermo Fisher Scientific, Inc.) solution. After 8 weeks of treatment, the lenses of the rats were observed, and the rats were then euthanized with an intraperitoneal injection of a lethal dose of pentobarbital sodium at $150 \mathrm{mg} / \mathrm{kg}$; death was confirmed with no heartbeat and respiration. The eyeballs were collected and fixed with $4 \%$ paraformaldehyde for $24 \mathrm{~h}$. The harvested retinal tissues were immersed in $10 \%$ formalin buffer.

Immunofluorescence evaluation of the status of transplanted cells. The status of transplanted cells in the ATRA and UC-MSCs groups was evaluated by immunofluorescence staining. Following fixation in $10 \%$ formalin buffer for $48 \mathrm{~h}$, the retinal tissues were embedded in paraffin and sliced at a thickness of $4 \mu \mathrm{m}$, followed by dewaxing with water. The tissues were then stained with 4',6-diamidino-2-phenylindole (DAPI), and examined under a fluorescence microscope (Leica, Microsystems GmbH).

Hematoxylin and eosin (H\&E) staining. Following fixation in $10 \%$ formalin buffer for $48 \mathrm{~h}$, the retinal tissues were embedded in paraffin and sliced at a thickness of $4 \mu \mathrm{m}$, followed by dewaxing with water. The sections were stained in hematoxylin solution (Bioswamp, Myhalic Biotechnology Co., Ltd.) for $3 \mathrm{~min}$ and subsequently in eosin solution (Bioswamp, Myhalic Biotechnology Co., Ltd.) for $3 \mathrm{~min}$ at room temperature. Following dehydration, the tissues were cleared with xylene and mounted with neutral balsam. After staining, the sections were observed under an upright microscope (Leica, Microsystems GmbH), and Leica Application Suite was used to collect and analyze the relevant parts of the samples.

Terminal deoxynucleotidyl transferase dUTP nick-end labeling (TUNEL) staining. Qualitative analysis of apoptosis in retinal tissues was performed using a TUNEL kit (Roche Diagnosticis) according to the manufacturer's instructions. Following fixation in $10 \%$ formalin buffer for $48 \mathrm{~h}$, the retinal tissues were embedded in paraffin and sliced at a thickness of 2-3 $\mu \mathrm{m}$, followed by dewaxing with water. The tissues were treated with protease $\mathrm{K}$ at room temperature for $30 \mathrm{~min}, 50 \mu \mathrm{l}$ of TUNEL reaction mixture in a dark humidified chamber at $37^{\circ} \mathrm{C}$ for $1 \mathrm{~h}$, and $50 \mu \mathrm{l}$ of converter-POD for $1 \mathrm{~h}$. Finally, diaminobenzidine was added, and the core was counterstained with hematoxylin. The sections were observed under an upright microscope (Leica, Microsystems $\mathrm{GmbH}$ ). The obtained images were quantified using Image Pro-Plus 6.0 (Media Cybernetics, Inc.).

Western blot analysis. The protein expression of CTGF, tissue plasminogen activator (t-PA), plasminogen activator inhibitor (PAI), transforming growth factor- $\beta 2$ (TGF $\beta 2$ ), and VEGF 
in retinal tissues was measured by western blot analysis. The collected retinas were homogenized in radioimmunoprecipitation assay lysis buffer (Bioswamp, Myhalic Biotechnology Co., Ltd.) and centrifuged at 12,000 x g for $15 \mathrm{~min}$. The concentration of proteins was detected using a bicinchoninic acid protein assay kit (Bioswamp, Myhalic Biotechnology Co., Ltd.). Total proteins $(20 \mu \mathrm{g})$ were separated by $10 \%$ sodium dodecyl sulfate-polyacrylamide gel electrophoresis and transferred onto polyvinylidene fluoride membranes (MilliporeSigma). The membranes were blocked with 5\% skim milk for $2 \mathrm{~h}$ at room temperature in Tris-buffered saline and incubated with primary antibodies against CTGF (1:1,000, ab6992, Abcam), t-PA (1:2,000, ab227069, Abcam), PAI (1:1,000, ab66705, Abcam), TGF 32 (1:1,000, ab113670, Abcam), VEGF (1:2,000, ab32152, Abcam), or GAPDH (1:1,000, 2118, Cell Signaling Technology, Inc.) for $1 \mathrm{~h}$ at room temperature, followed by incubation with goat anti-rabbit IgG secondary antibody (1:10,000, SAB43714, Bioswamp, Myhalic Biotechnology Co., Ltd.) for $2 \mathrm{~h}$ at room temperature. Immunoreactivity was visualized by colorimetric reaction using an enhanced chemiluminescence substrate buffer (MilliporeSigma). Membranes were scanned with a Tanon-5200 imager (Shanghai Tianneng Technology Co., Ltd.) and analyzed using IQTL 8.1 software.

Enzyme-linked immunosorbent assay (ELISA). The levels of interleukin (IL)-1 (RA20108), IL-6 (RA20607) and IL-10 (RA20090) in the serum of retinas were detected using ELISA kits (Bioswamp, Myhalic Biotechnology Co., Ltd.) according to the manufacturer's instructions. The absorbance was measured at $450 \mathrm{~nm}$ using a microplate reader (Labsystems Multiskan MS, Finland).

Statistical analysis. The data of the present study are expressed as the means \pm standard deviation (SD). Statistical analysis was performed using IBM SPSS statistics 19.0. The statistical significance of differences between $>2$ groups was evaluated by one-way analysis of variance followed by Bonferroni's test and significant differences between 2 groups were analyzed using a t-test. $\mathrm{P}<0.05$ was considered to indicate a statistically significant difference.

\section{Results}

Successful extraction of UC-MSCs. To confirm that UC-MSCs were successfully extracted, cells were visually evaluated using a microscope, whereby the typical spindle-shaped fibroblast-like morphology of UC-MSCs was observed (Fig. 1A). Flow cytometry was then carried out to detect the expression of surface antigens of UC-MSCs. As shown in Fig. 1B, the extracted cells strongly expressed the mesenchymal cell markers, CD90 (97.37\%), CD44 (96.58\%), CD29 (96.82\%), CD73 (97.58\%) and CD105 (97.98\%), while the hematopoietic markers, HLA-DR (1.80\%), CD45 (1.24\%), and CD34, (1.72\%) were absent $(20,21)$. These findings indicate that UC-MSCs were successfully isolated without the inclusion of other cell populations.

ATRA promotes UC-MSC proliferation and differentiation. Following treatment of the UC-MSCs with ATRA for 24, 48 and $72 \mathrm{~h}$, cell proliferation and differentiation were determined. Compared to the untreated cells, the viability of the ATRA-treated cells significantly increased ( $\mathrm{P}<0.01$, Fig. 2A). In addition, ATRA exerted minimal effects on the apoptosis of UC-MSCs (Fig. 2B). Immunofluorescence staining was performed to examine the differentiation of UC-MSCs treated with ATRA. Strong positive staining was observed for the glial cell marker, GFAP, the nerve cell marker, Nestin, and the neuron-specific marker, NSE (26), in the ATRA-treated UC-MSCs compared to that of signals in the untreated UC-MSCs (Fig. 2C). In addition, the mRNA expression levels of Nestin, NSE and GFAP in the untreated UC-MSCs were lower than those in the ATRA-treated UC-MSCs (Fig. 2D), suggesting that ATRA promoted UC-MSC differentiation into different types of nerve cells. Moreover, reticular neuron-like cells were observed (red boxes) after the UC-MSCs were treated with ATRA for $24 \mathrm{~h}$ (Fig. 2C). Thus, $24 \mathrm{~h}$ of ATRA treatment was selected as the final treatment duration for UC-MSCs in the transplantation experiment.

Transplantation of ATRA-treated or untreated UC-MSCs attenuates $S T Z$-induced DR in rats. The blood glucose levels of all selected rats with STZ-induced DR were $>16.7 \mathrm{mmol} / \mathrm{l}$ (data not shown). At 8 weeks following transplantation with CM-Dil-labeled UC-MSCs, the UC-MSCs in retinal tissue were visualized by immunofluorescence. A shown in Fig. 3A, retinal tissues contained a large number of well-distributed UC-MSCs (white arrow pointing towards red area). The transplantation of untreated or ATRA-treated UC-MSCs improved the lens opacity of rats with DR macroscopically (Fig. 3B).

Furthermore, the pathological morphology of retinal tissues was investigated by H\&E staining (Fig. 4). The surface of the retina was smooth, and cells at the outer nuclear layer (ONL, upward arrow), inner nuclear layer (INL, downward arrow) and retinal ganglion cells (RGCs) were all regularly arranged in the CON group. However, cells at the ONL and INL appeared in irregular arrangements in the MOD group. Additionally, RGCs protruded through the inner limiting membrane with a rough retinal surface (red arrows). With UC-MSC treatment, the cells at the ONL and INL exhibited a regular arrangement. This was accompanied by greater RGC protrusion, which was even more prominent in the ATRA group and closely resembled that of the CON group. These findings suggest that UC-MSC transplantation attenuated DR in rats and that ATRA further accentuated the effects of UC-MSCs.

Transplantation of untreated or ATRA-treated UC-MSCs attenuates apoptosis in retinal tissue of rats with DR. TUNEL assay was performed to detect apoptosis in retinal tissues (Fig. 5). The number of TUNEL-positive cells (brown) was significantly higher in the MOD group compared to the CON group $(\mathrm{P}<0.01)$. However, the number of TUNEL-positive cells in the UC-MSC group decreased compared to the MOD group $(\mathrm{P}<0.01)$ and further decreased in the ATRA group $(\mathrm{P}<0.01)$.

Transplantation of untreated or ATRA-treated UC-MSCs inhibits neovascularization and imminent fibrosis in retinal tissue of rats with DR. The expression levels of the angiogenesis-related proteins, VEGF, t-PA and PAI, and that of the fibrosis-associated proteins, CTGF and TGF 32 , were assessed 

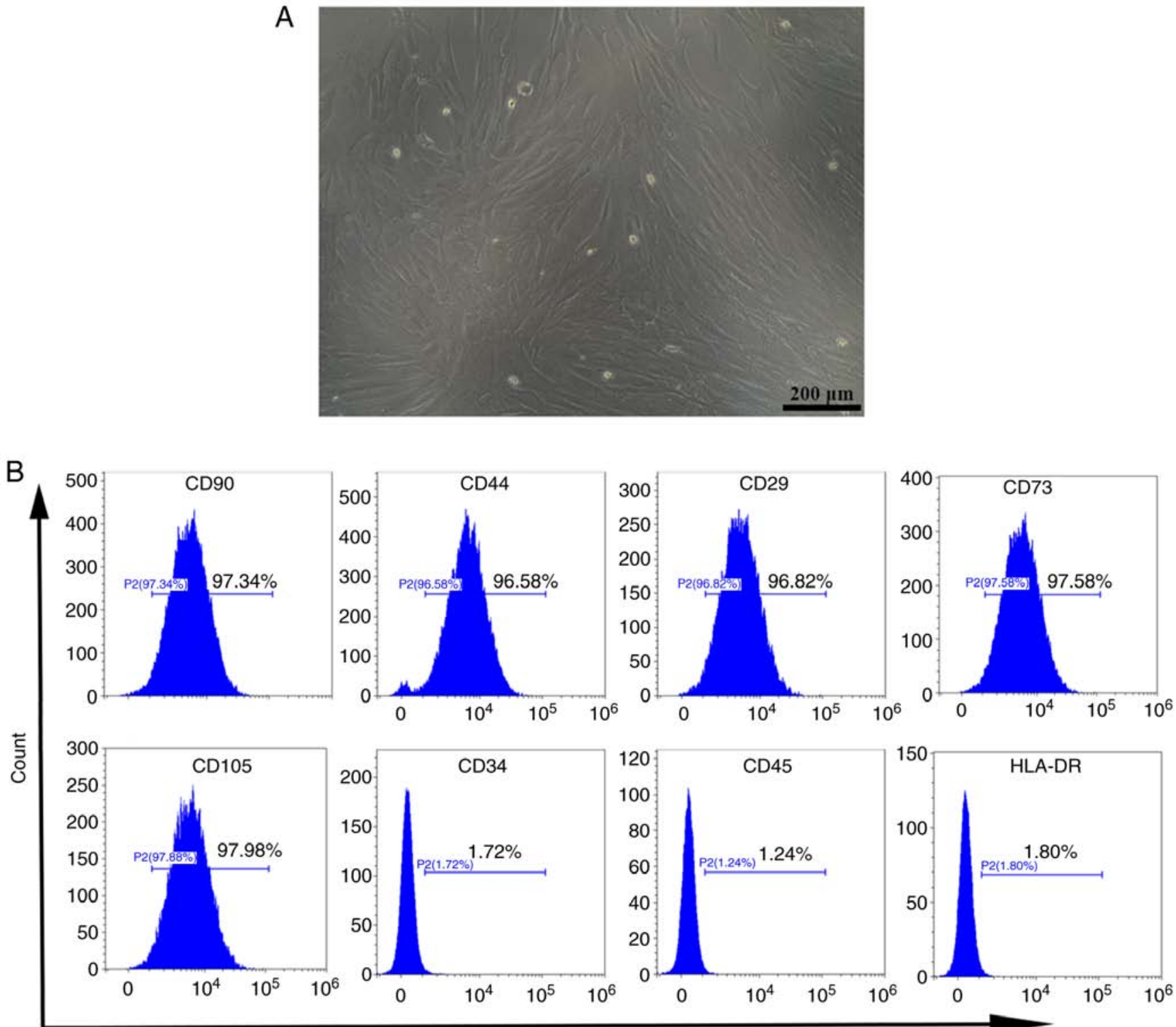

FITC

Figure 1. Characterization of UC-MSCs. (A) Morphology of UC-MSCs (magnification, x100). Scale bar, $200 \mu \mathrm{m}$. (B) Expression of surface antigens of UC-MSCs. UC-MSCs, umbilical cord-derived mesenchymal stem cells.

by western blot analysis. As shown in Fig. 6, the levels of all the above-mentioned proteins were upregulated in the MOD group compared to those in the $\mathrm{CON}$ group $(\mathrm{P}<0.01)$. Comparatively, UC-MSC transplantation decreased the expression of these proteins $(\mathrm{P}<0.01)$ and this decrease was further enhanced in the ATRA group compared to the UC-MSC group $(\mathrm{P}<0.01$, apart from t-PA). Therefore, UC-MSC transplantation inhibited the neovascularization of retinal tissues in rats with DR, and treatment of UC-MSCs with ATRA further promoted this inhibition.

Transplantation of untreated or ATRA-treated UC-MSCs attenuates inflammation in retinal tissue of rats with $D R$. As shown in Fig. 7, compared to the CON group, the secretion levels of IL- 6 and IL- 1 in the serum of retinas were notably increased in the MOD group $(\mathrm{P}<0.01)$. In addition, compared to the MOD group, the levels of IL- 6 and IL-1 were markedly decreased in the UC-MSC group $(\mathrm{P}<0.01)$ and were even further decreased in the ATRA group $(\mathrm{P}<0.01)$. The expression IL-10 exhibited an opposite trend to that of IL-6 and IL-1, indicating that UC-MSC transplantation attenuated inflammation in the retinal tissue of rats with DR, which was further suppressed by treatment of the UC-MSCs with ATRA.

\section{Discussion}

MSC transplantation is considered a promising intervention therapy for DR $(1,27)$. Differentiation into nerve cells is one of the vital functions of MSCs in DR treatment $(11,13,28)$. Neural stem cells derived from UC-MSCs have been shown to exert a neuroprotective effect and to notably attenuate the progression of DR (13). Nevertheless, research into the role of MSCs in the treatment of DR remains incomplete, and the mechanisms of retinal repair at the molecular level remain unclear. The present study demonstrated that treatment with ATRA promoted the proliferation of UC-MSCs and their differentiation into 


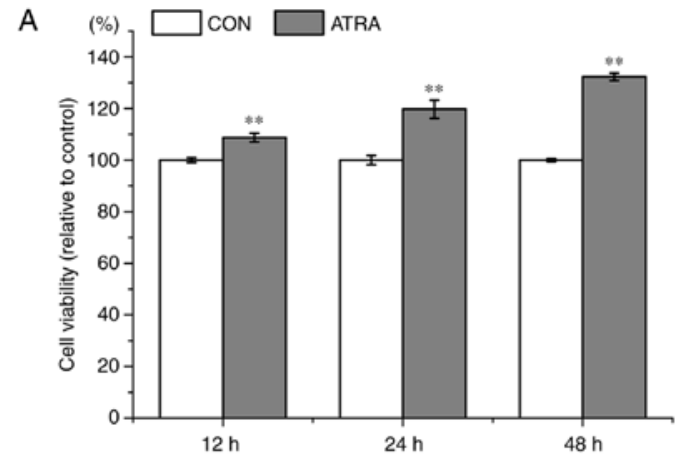

B

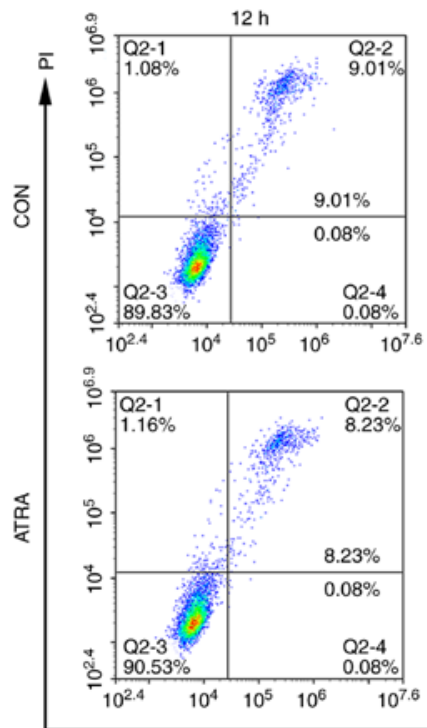

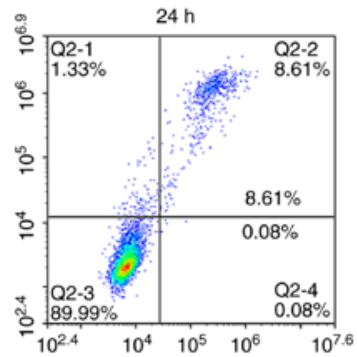
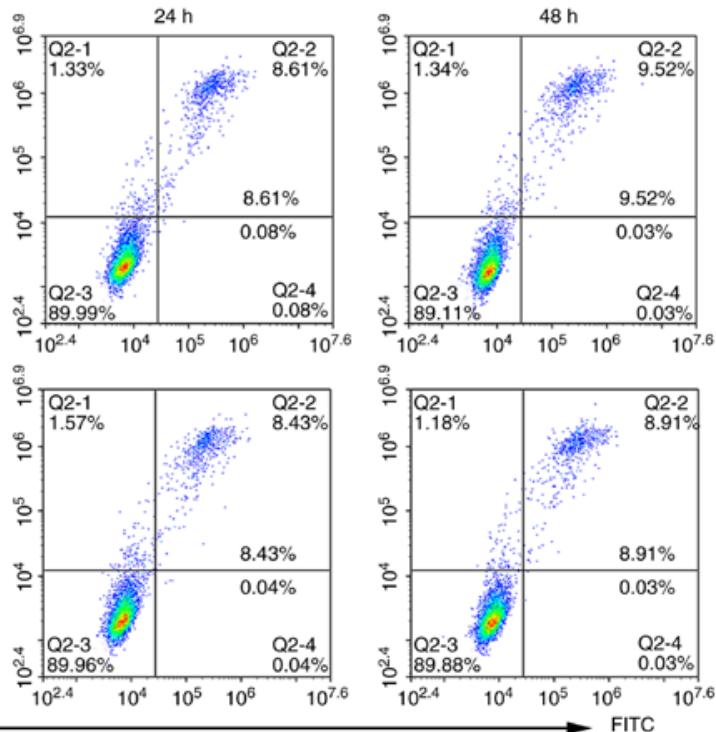

C
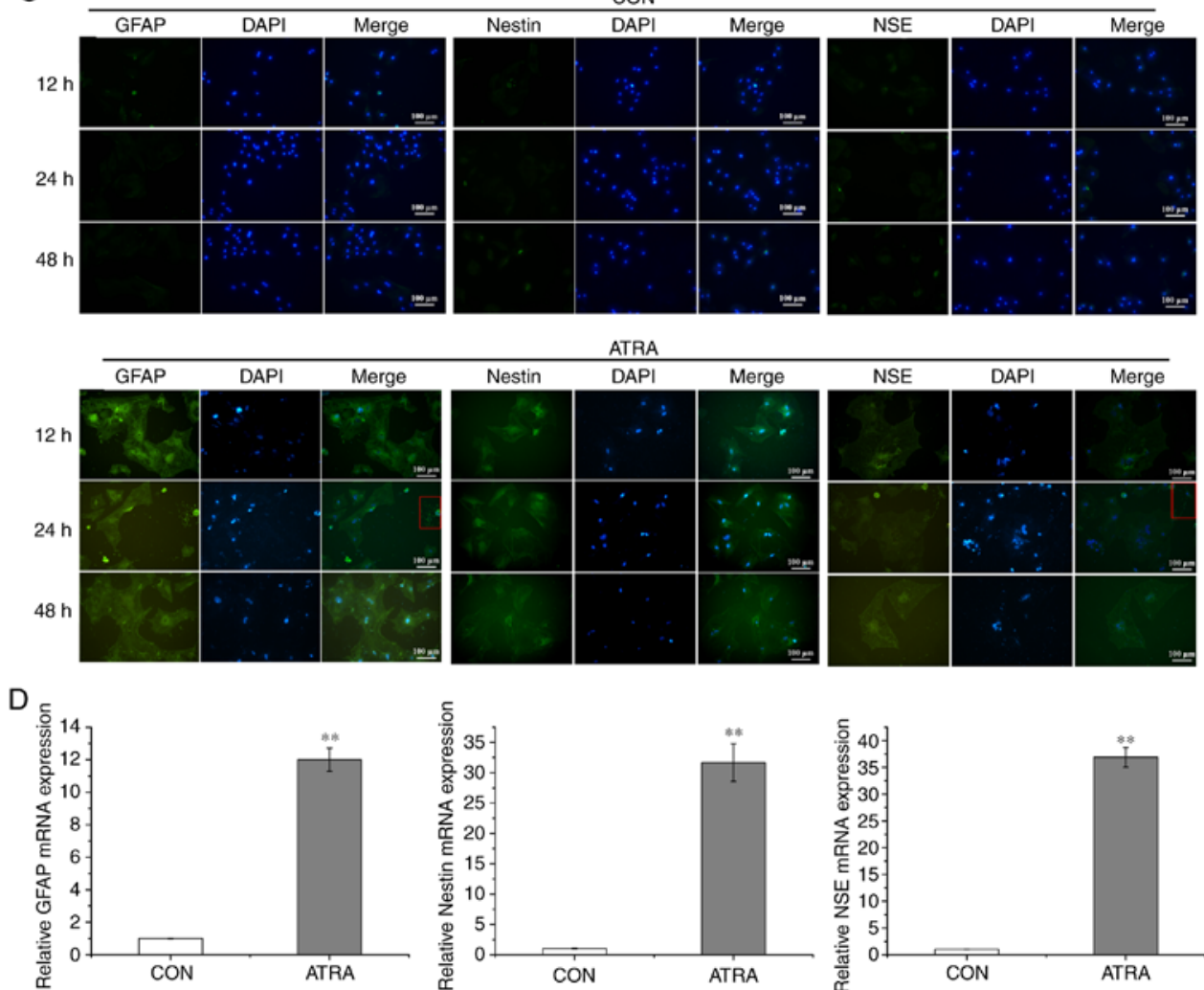

Figure 2. Effect of ATRA on UC-MSC behavior. (A) Cell viability and (B) apoptosis of UC-MSCs with or without ATRA treatment. (C) Immunofluorescence of GFAP, Nestin and NSE in ATRA-treated/untreated UC-MSCs (magnification, x200). Scale bar, $100 \mu \mathrm{m}$. (D) mRNA expression of GFAP, Nestin and NSE in ATRA-treated/untreated UC-MSCs. Data represent the means $\pm \mathrm{SD}(\mathrm{n}=3) ;{ }^{* *} \mathrm{P}<0.01$ compared to CON. ATRA means ATRA-treated UC-MSCs. ATRA, all-trans retinoic acid; UC-MSCs, umbilical cord-derived mesenchymal stem cells; GFAP, glial fibrous acidic protein; NSE, Neuron-specific enolase. 
A
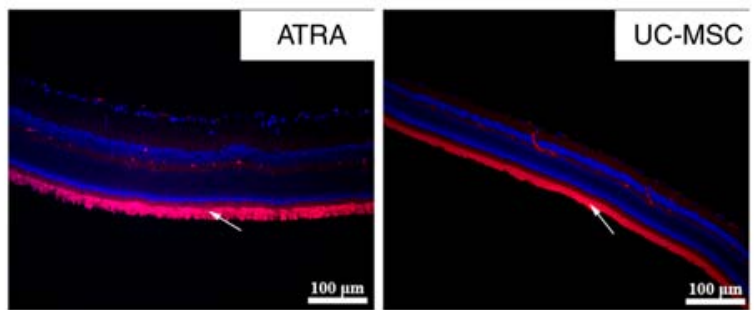

B
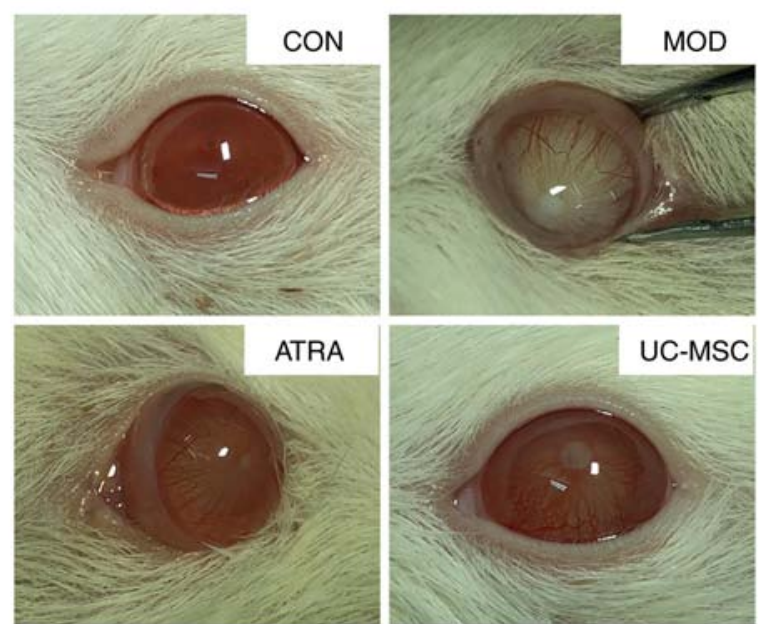

Figure 3. Effect of UC-MSCs on DR. (A) Distribution of CM-Dil-labeled UC-MSCs (the pink line of the white arrow) in retinal tissue (magnification, 200). Scale bar, $100 \mu \mathrm{m}$. The pink line denotes CM-Dil-labeled UC-MSCs and the blue lines denotes DAPI-labeled cell nuclei in retinal tissue. (B) Comparison of lens of rats subjected to various treatments. UC-MSCs, umbilical cord-derived mesenchymal stem cells (untreated); DR, diabetic retinopathy; ATRA, UC-MSCs treated with all-trans retinoic acid; MOD, model group with DR; CON, control.

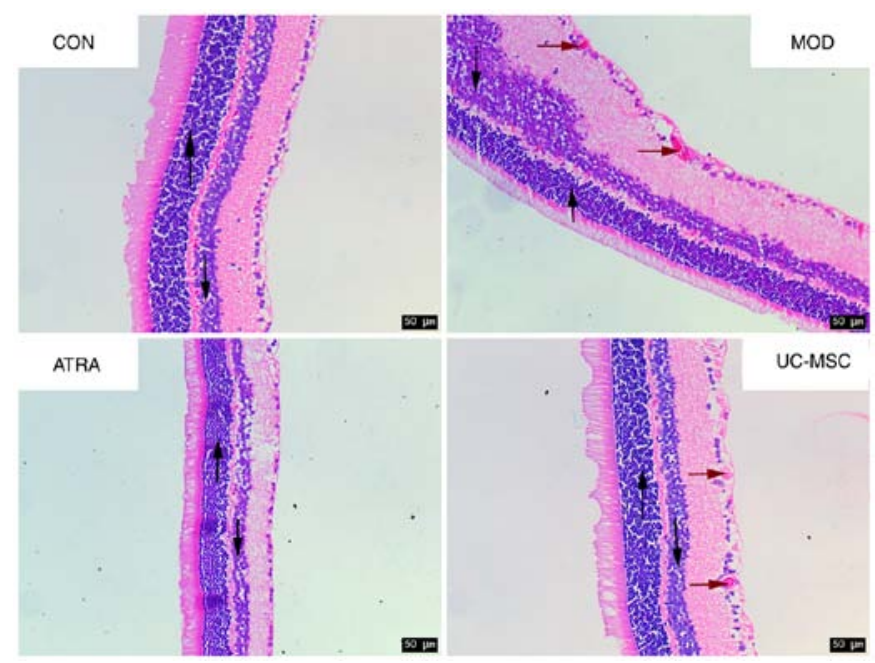

Figure 4. Pathological observation of retinal tissue. Downward arrows indicate inner nuclear layer; upward arrows indicate outer nuclear layer; red arrows indicated RGCs protruding through the inner limiting membrane with a rough retinal surface. Scale bar, $50 \mu \mathrm{m}$. UC-MSCs, umbilical cord-derived mesenchymal stem cells (untreated); DR, diabetic retinopathy; ATRA, UC-MSCs treated with all-trans retinoic acid; MOD, model group with DR; CON, control.

nerve cells. The transplantation of UC-MSCs with or without ATRA treatment visually ameliorated DR in rats with STZ-induced diabetes, alleviated retinal tissue damage and
A

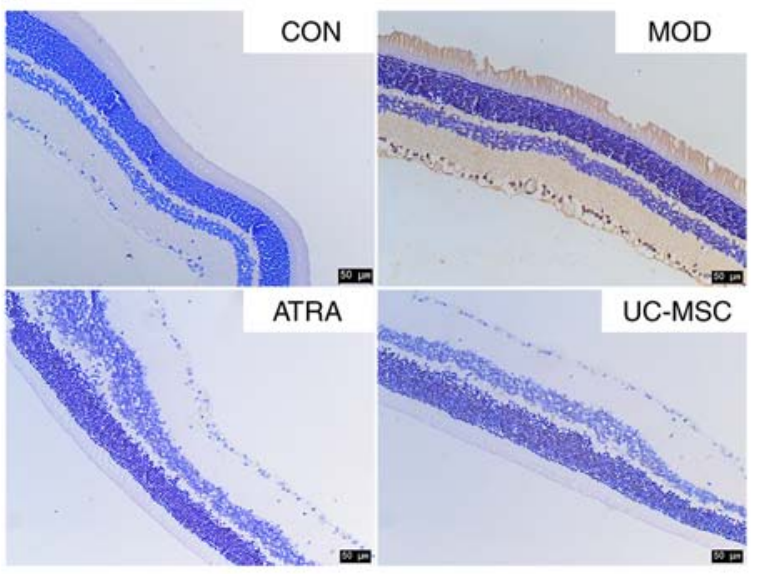

B

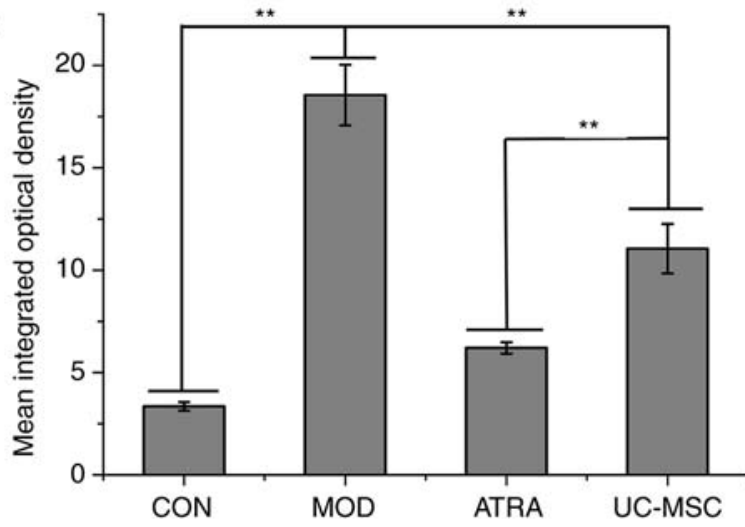

Figure 5. (A) Apoptosis detection in retinal tissue using TUNEL staining and (B) mean integrated optical density quantification of TUNEL-positive cells evaluation using Image Pro-Plus 6.0. Data represent the means \pm SD $(n=3)$, ${ }^{* *} \mathrm{P}<0.01$. Scale bar, $50 \mu \mathrm{m}$. Brown stained cells in (A) denote TUNEL-positive cells (apoptotic). UC-MSCs, umbilical cord-derived mesenchymal stem cells (untreated); DR, diabetic retinopathy; ATRA, UC-MSCs treated with all-trans retinoic acid; MOD, model group with DR; CON, control.

apoptosis, inhibited angiogenesis and attenuated inflammation in the retina via the regulation of various cytokines. Moreover, UC-MSCs treated with ATRA exerted a more prominent therapeutic effect than the transplantation of untreated UC-MSCs.

Several experimental and clinical studies have identified abnormal ocular angiogenesis as a critical pathophysiologic mediator of DR (29-31); for example, uncontrolled ocular angiogenesis leads to a leaky and fragile vasculature. The subsequent accumulation of fluids and protein exudates in ocular cavities results in the enhancement of corneal opacity, which eventually leads to blindness, a devastating feature of the final stage of the disease (32). VEGF, a recognized pro-angiogenic cytokine, is overexpressed in response to a maintained hyperglycemic environment $(33,34)$, resulting in retinal neovascularization, which is involved in the pathogenesis of DR (35). Anti-VEGF therapy has been considered safe and promising for the management of DR (35). Apart from activating retinal angiogenesis, VEGF participates in activating several biologically active substances, such as t-PA and PAI, which are involved in neovascularization (36). T-PA and PAI are highly expressed in DR and are associated with their occurrence and development $(36,37)$. The present study demonstrated that VEGF, t-PA and PAI were highly expressed in rats with DR, but were downregulated after UC-MSC transplantation, resulting in the attenuation of 

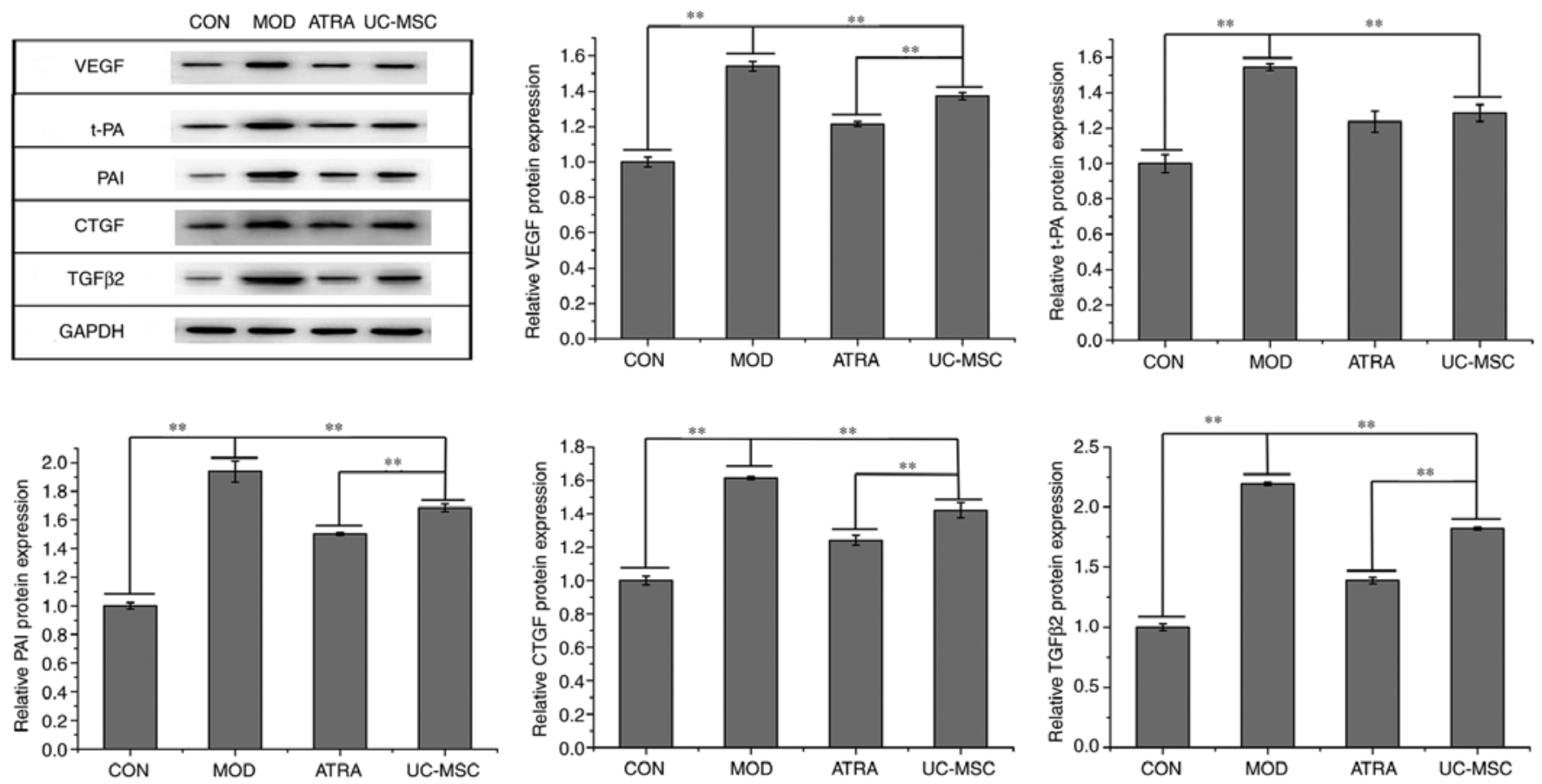

Figure 6. Angiogenesis and fibrosis in retinal tissues. Expression and quantification of proteins related to angiogenesis- and fibrosis-related proteins in retinal tissue. Data represent the means $\pm \mathrm{SD}(\mathrm{n}=3),{ }^{* *} \mathrm{P}<0.01$. VEGF, vascular endothelial growth factor; t-PA, tissue plasminogen activator; PAI, plasminogen activator inhibitor; TGF $\beta 2$, transforming growth factor $\beta 2$; CTGF, connective tissue growth factor; UC-MSCs, umbilical cord-derived mesenchymal stem cells (untreated); DR, diabetic retinopathy; ATRA, UC-MSCs treated with all-trans retinoic acid; MOD, model group with DR; CON, control.

DR. In addition, UC-MSCs treated with ATRA exhibited a better efficiency than untreated UC-MSCs. Retinal fibrosis is another important factor in the pathogenesis of DR. It has been reported that elevated levels of CTGF and TGF $\beta 2$ are positively associated with the formation of retinal fibrosis (38-40), and that fibrosis is accelerated by the upregulation of CTGF and TGFß2 in DR (41). The intravitreal injection of anti-CTGF shRNA has been shown to reduce the level of CTGF in diabetic mice, leading to decreased injury in the retinal microvascular structure (42). The present study demonstrated that UC-MSC transplantation reduced the expression $\omega$ of CTGF and TGF $\beta 2$ in rats with DR.

Additionally, diabetic-induced inflammation plays a vital role in the pathogenesis of DR, which may induce leukocyte-endothelial interactions and eventually cause damage to the retinal microvasculature $(22,43)$. MSCs possess immunomodulatory functions and are considered to be promising alternative agents in the treatment of inflammatory diseases (44). MSCs infected with C-X-C chemokine receptor type 4 have been shown to attenuate the progression of DR by inhibiting the expression of inflammation-regulating cytokines, such as IL-6 and tumor necrosis factor- $\alpha$ (8). The present study demonstrated that UC-MSC transplantation reduced the levels of pro-inflammatory IL-1 and IL-6 (45), while increasing an anti-inflammatory response (46) in rats with STZ-induced DR. Moreover, the ATRA-treated UC-MSCs exhibited a better efficiency than the untreated UC-MSCs.

Overall, the present study suggested that UC-MSCs attenuated STZ-induced DR in rats by regulating cytokines related to angiogenesis and inflammation at the molecular level. The effect of angiogenesis and anti-apoptosis may be associated with the differentiation of MSCs into neural cells, although

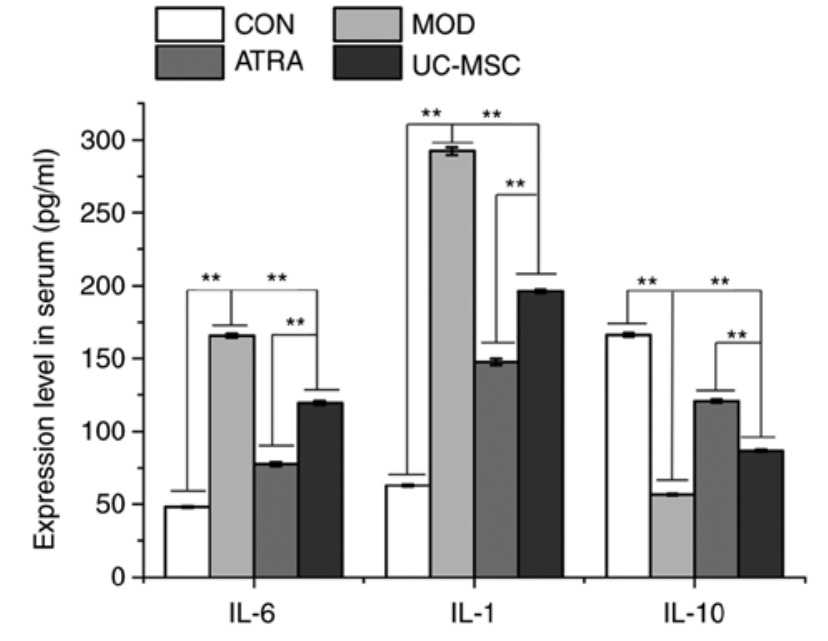

Figure 7. Levels of IL-1, IL-6 and IL-10 in the serum of retinas. Data represent the means $\pm \mathrm{SD}(\mathrm{n}=3),{ }^{* *} \mathrm{P}<0.01$. UC-MSCs, umbilical cord-derived mesenchymal stem cells (untreated); DR, diabetic retinopathy; ATRA, UC-MSCs treated with all-trans retinoic acid; MOD, model group with DR; CON, control.

the effect may also be in conjunction with the differentiation of MSCs into neural cells. The transplantation of UC-MSCs treated with ATRA displayed a more prominent therapeutic effect than untreated UC-MSCs, as demonstrated by its better regulation of angiogenesis and inflammation in rats with STZ-induced DR, and the proliferation and differentiation of MSCs. These findings provide a theoretical basis for and new insight into the application of MSCs in the treatment of DR. However, due to neglect, the weights of the rats were not recorded in the present study. The authors aim to certainly 
record the weight changes of rats in follow-up studies, which will explore the specific signaling pathways associated with angiogenesis and the inflammatory response in rats with DR treated with UC-MSCs.

\section{Acknowledgements}

Not applicable.

\section{Funding}

This study was supported by the Health Commission of Hubei Province Scientific Research Project (grant no. WJ2009F038).

\section{Availability of data and materials}

The datasets used and/or analyzed during the current study are available from the corresponding author on reasonable request.

\section{Authors' contributions}

$\mathrm{XX}$ and ZC were responsible for the conception and design of the study. KZ, JL, GD, PW and HX performed the experiments. $\mathrm{KZ}$ and JL analyzed the data and drafted the manuscript. XX and $\mathrm{ZC}$ revised the manuscript. All authors read and approved the final manuscript.

\section{Ethics approval and consent to participate}

All animal protocols were approved by the Institutional Review Board of Wuhan Myhalic Biotechnology Co., Ltd. based on the ethical Guidelines for Animal Care and Use of the Model Animal Research Institute (HLK-20180802-01).

\section{Patient consent for publication}

Not applicable.

\section{Competing interests}

The authors declare that they have no competing interests.

\section{References}

1. Fiori A, Terlizzi V, Kremer H, Gebauer J, Hammes HP, Harmsen MC and Bieback K: Mesenchymal stromal/stem cells as potential therapy in diabetic retinopathy. Immunobiology 223 729-743, 2018

2. Whiting DR, Guariguata L, Weil C and Shaw J: IDF diabetes atlas: Global estimates of the prevalence of diabetes for 2011 and 2030. Diabetes Res Clin Pract 94: 311-321, 2011

3. Yau JW, Rogers SL, Kawasaki R, Lamoureux EL, Kowalski JW, Bek T, Chen SJ, Dekker JM, Fletcher A, Grauslund J, et al: Global prevalence and major risk factors of diabetic retinopathy. Diabetes Care 35: 556-564, 2012.

4. Selvaraj K, Gowthamarajan K, Karri VV, Barauah UK, Ravisankar V and Jojo GM: Current treatment strategies and nanocarrier based approaches for the treatment and management of diabetic retinopathy. J Drug Target 25: 386-405, 2017.

5. Zhang $\mathrm{C}, \mathrm{Xu} \mathrm{Y}$, Tan HY, Li S, Wang N, Zhang Y and Feng Y Neuroprotective effect of He-Ying-Qing-Re formula on retinal ganglion cell in diabetic retinopathy. J Ethnopharmacol 214 179-189, 2018.

6. Ding DC, Shyu WC and Lin SZ: Mesenchymal stem cells. Cell Transplant 20: 5-14, 2011
7. Nagamura-Inoue $\mathrm{T}$ and $\mathrm{He} \mathrm{H}$ : Umbilical cord-derived mesenchymal stem cells: Their advantages and potential clinical utility. World J Stem Cells 6: 195-202, 2014.

8. Gu X, Yu X, Zhao C, Duan P, Zhao T, Liu Y, Li S, Yang Z, Li Y, Qian C, et al: Efficacy and safety of autologous bone marrow mesenchymal stem cell transplantation in patients with diabetic retinopathy. Cell Physiol Biochem 49: 40-52, 2018.

9. Wang J, Zhang W, He GH, Wu B and Chen S: Transfection with CXCR4 potentiates homing of mesenchymal stem cells in vitro and therapy of diabetic retinopathy in vivo. Int J Ophthalmol 11: 766-772, 2018

10. Scalinci SZ, Scorolli L, Corradetti G, Domanico D, Vingolo EM, Meduri A, Bifani M and Siravo D: Potential role of intravitreal human placental stem cell implants in inhibiting progression of diabetic retinopathy in type 2 diabetes: Neuroprotective growth factors in the vitreous. Clin Ophthalmol 5: 691-696, 2011.

11. Yang Z, Li K, Yan X, Dong F and Zhao C: Amelioration of diabetic retinopathy by engrafted human adipose-derived mesenchymal stem cells in streptozotocin diabetic rats. Graefes Arch Clin Exp Ophthalmol 248: 1415-1422, 2010.

12. Hsieh JY, Wang HW, Chang SJ, Liao KH, Lee IH, Lin WS, Wu CH, Lin WY and Cheng SM: Mesenchymal stem cells from human umbilical cord express preferentially secreted factors related to neuroprotection, neurogenesis, and angiogenesis. PLoS One 8: e72604, 2013.

13. Zhang W, Wang Y, Kong J, Dong M, Duan H and Chen S: Therapeutic efficacy of neural stem cells originating from umbilical cord-derived mesenchymal stem cells in diabetic retinopathy. Sci Rep 7: 408, 2017.

14. Mottaghi S, Larijani B and Sharifi AM: Atorvastatin: An efficient step forward in mesenchymal stem cell therapy of diabetic retinopathy. Cytotherapy 15: 263-266, 2013.

15. Siddikuzzaman, Guruvayoorappan $\mathrm{C}$ and Berlin Grace VM: All trans retinoic acid and cancer. Immunopharmacol Immunotoxicol 33: 241-249, 2011.

16. Gong M, Bi Y, Jiang W, Zhang Y, Chen L, Hou N, Chen J and Li T: Retinoic acid receptor beta mediates all-trans retinoic acid facilitation of mesenchymal stem cells neuronal differentiation. Int J Biochem Cell Biol 45: 866-875, 2013.

17. Tanaka K, Tamiya-Koizumi K, Hagiwara K, Ito H, Takagi A, Kojima T, Suzuki M, Iwaki S, Fujii S, Nakamura M, et al: Role of down-regulated neutral ceramidase during all-trans retinoic acid-induced neuronal differentiation in SH-SY5Y neuroblastoma cells. J Biochem 151: 611-620, 2012.

18. Murashov AK, Pak ES, Hendricks WA, Owensby JP, Sierpinski PL, Tatko LM and Fletcher PL: Directed differentiation of embryonic stem cells into dorsal interneurons. FASEB J 19: 252-254, 2005.

19. Xi J and Yang Z: Expression of RALDHs (ALDH1As) and CYP26s in human tissues and during the neural differentiation of P19 embryonal carcinoma stem cell. Gene Expr Patterns 8: 438-442, 2008.

20. Pourjafar M, Saidijam M, Etemadi K and Najafi R: All-trans retinoic acid enhances in vitro mesenchymal stem cells migration by targeting matrix metalloproteinases 2 and 9. Biotechnol Lett 39: 1263-1268, 2017.

21. Jin $\mathrm{W}, \mathrm{Xu} Y \mathrm{P}$, Yang $\mathrm{AH}$ and Xing YQ: In vitro induction and differentiation of umbilical cord mesenchymal stem cells into neuron-like cells by all-trans retinoic acid. Int J Ophthalmol 8: 250-256, 2015.

22. Yin Y, Chen F, Wang W, Wang $\mathrm{H}$ and Zhang $\mathrm{X}$ : Resolvin D1 inhibits inflammatory response in STZ-induced diabetic retinopathy rats: Possible involvement of NLRP3 inflammasome and NF- $\kappa \mathrm{B}$ signaling pathway. Mol Vis 23: 242-250, 2017.

23. Jenkins AJ, Joglekar MV, Hardikar AA, Keech AC, O'Neal DN and Januszewski AS: Biomarkers in diabetic retinopathy. Rev Diabet Stud 12: 159-195, 2015.

24. Livak KJ and Schmittgen TD: Analysis of relative gene expression data using real-time quantitative PCR and the 2(-Delta Delta C(T)) method. Methods 25: 402-408, 2001.

25. Xie X, Peng J, Huang K, Huang J, Shen X, Liu P and Huang H: Polydatin ameliorates experimental diabetes-induced fibronectin through inhibiting the activation of NF- $\kappa \mathrm{B}$ signaling pathway in rat glomerular mesangial cells. Mol Cell Endocrinol 362: 183-193, 2012.

26. Fu X, Li S, Zhou S, Wu Q, Jin F and Shi J: Stimulatory effect of icariin on the proliferation of neural stem cells from rat hippocampus. BMC Complement Altern Med 18: 34, 2018.

27. Ding SSL, Subbiah SK, Khan MSA, Farhana A and Mok PL: Empowering mesenchymal stem cells for ocular degenerative disorders. Int J Mol Sci 20: pii: E1784, 2019. 
28. Yue X, Zhifeng G, Biyu S, Guofeng X, Tianqiu Z, Jinxia J, Jing X, Suzhe L, Man L, Wei T, et al: Roles of Wnt $/ \beta$-catenin signaling in retinal neuron-like differentiation of bone marrow mesenchymal stem cells from nonobese diabetic mice. J Mol Neurosci 49: 250-261, 2013.

29. $\mathrm{Wu} \mathrm{W}$, Lei $\mathrm{H}$, Shen $\mathrm{J}$ and Tang L: The role of netrin-1 in angiogenesis and diabetic retinopathy: A promising therapeutic strategy. Discov Med 23: 315-323, 2017.

30. Gardlik R and Fusekova I: Pharmacologic therapy for diabetic retinopathy. Semin Ophthalmol 30: 252-263, 2015.

31. Khan AA, Rahmani AH and Aldebasi YH: Diabetic retinopathy: Recent updates on different biomarkers and some therapeutic agents. Curr Diabetes Rev 14: 523-533, 2018.

32. Zhang SX and Ma JX: Ocular neovascularization: Implication of endogenous angiogenic inhibitors and potential therapy. Prog Retin Eye Res 26: 1-37, 2007.

33. Boyer DS, Hopkins JJ, Sorof J and Ehrlich JS: Anti-vascular endothelial growth factor therapy for diabetic macular edema. Ther Adv Endocrinol Metab 4: 151-169, 2013.

34. Stewart MW: Anti-vascular endothelial growth factor drug treatment of diabetic macular edema: The evolution continues. Curr Diabetes Rev 8: 237-246, 2012.

35. Bahrami B, Hong T, Gilles MC and Chang A: Anti-VEGF therapy for diabetic eye diseases. Asia Pac J Ophthalmol (Phila) 6: 535-545, 2017.

36. Wu SL, Zhan DM, Xi SH and He XL: Roles of tissue plasminogen activator and its inhibitor in proliferative diabetic retinopathy. Int J Ophthalmol 7: 764-767, 2014.

37. Wu S and He X: The correlation study of the expression of VEGF with t-PA and PAI expression in plasma and intraocular tissue in proliferative diabetic retinopathy. Zhonghua Yan Ke Za Zh 50: 448-453, 2014 (In Chinese).

38. Zhang M, Chu S, Zeng F and Xu H: Bevacizumab modulates the process of fibrosis in vitro. Clin Exp Ophthalmol 43: 173-179, 2015.
39. Van Geest RJ, Lesnik-Oberstein SY, Tan HS, Mura M, Goldschmeding R, Van Noorden CJ, Klaassen I and Schlingemann RO: A shift in the balance of vascular endothelial growth factor and connective tissue growth factor by bevacizumab causes the angiofibrotic switch in proliferative diabetic retinopathy. Br J Ophthalmol 96: 587-590, 2012.

40. Kuiper EJ, Van Nieuwenhoven FA, de Smet MD, van Meurs JC, Tanck MW, Oliver N, Klaassen I, Van Noorden CJ, Goldschmeding R and Schlingemann RO: The angio-fibrotic switch of VEGF and CTGF in proliferative diabetic retinopathy. PLoS One 3: e2675, 2008.

41. Zhang Q, Qi Y, Chen L, Shi X, Bai Y, Huang L, Yu W, Jiang Y, Zhao $\mathrm{M}$ and $\mathrm{Li} \mathrm{X}$ : The relationship between anti-vascular endothelial growth factor and fibrosis in proliferative retinopathy: Clinical and laboratory evidence. Br J Ophthalmol 100: 1443-1450, 2016.

42. Hu B, Zhang Y, Zeng Q, Han Q, Zhang L, Liu M and Li X: Intravitreal injection of ranibizumab and CTGF shRNA improves retinal gene expression and microvessel ultrastructure in a rodent model of diabetes. Int J Mol Sci 15: 1606-1624, 2014.

43. Cheung N, Mitchell P and Wong TY: Diabetic retinopathy. Lancet 376: 124-136, 2010.

44. Regmi S,Pathak S, Kim JO, Yong CS and Jeong JH: Mesenchymal stem cell therapy for the treatment of inflammatory diseases: Challenges, opportunities, and future perspectives. Eur J Cell Biol 98: 151041, 2019.

45. Navarro-Gonzalez JF and Mora-Fernandez C: The role of inflammatory cytokines in diabetic nephropathy. J Am Soc Nephrol 19: 433-442, 2008.

46. Petersen AM and Pedersen BK: The anti-inflammatory effect of exercise. J Appl Physiol (1985) 98: 1154-1162, 2005. 